(C) [2008] IEEE. Reprinted, with permission, from [Mao Lin Huang, Jie Liang and Quang Vinh Nguyen,A A Usability Study on the Use of Multi-Context Visualization, 2008, Fifth International Conference on Computer Graphics, Imaging and Visualisation, 2008]. This material is posted here with permission of the IEEE. Such ermission of the IEEE does not in any way imply IEEE endorsement of any of the University of Technology, Sydney's products or services. Internal or personal use of this material is permitted. However, permission to reprint/republish this material for advertising or promotional purposes or for creating new collective works for resale or redistribution must be obtained from the IEEE by writing to pubs-permissions@ieee.org. By choosing to view this document, you agree to all provisions of the copyright laws protecting it 


\title{
A Usability Study on the Use of Multi-Context Visualization
}

\author{
Mao Lin Huang, Jie Liang, Quang Vinh Nguyen \\ Faculty of Information Technology, University of Technology, Sydney \\ \{maolin@it.uts.edu.au,jiliang@it.uts.edu.au, quvnguye@it.uts.edu.au\}
}

\begin{abstract}
Graph visualization has been widely used in realworld applications, as it provides better presentation of overall data structure. However, there are navigation problems existing in deep and large relational datasets. To address these challenges, a new technique called multi-context visualization, which provides users with rich contextual information, has been proposed as the solution to the navigation in large scale datasets. This paper evaluates the multi-context visualization by conducting an experiment-based user study. To answer whether the more contextual information positively assist in making more accurate and easier decisions, it aims to evaluate the effectiveness and efficiency of the multicontext visualization, by measuring the user performance. Specifically, this usability test was designed to test if the use of multiple context views can improve navigation problems for deep and large relational data sets.
\end{abstract}

Keywords--- Usability Study, Evaluation, MultiContext, Information Visualization, Navigation.

\section{Introduction}

In almost every field, making precise decision becomes crucial. However, it is getting more difficult for people to analyse and make decision with large information, because more complex situations, dynamic data, and complicated tasks exceed human capacity to perceive, understand and reason. To make a profound difference, successful visual analytics tools enable to synthesize and simplify information and derive insights from massive data and provide timely assessments. Visual analytics is defined as the science of analytical reasoning facilitated by interactive visual interfaces [1]. It is a new interdisciplinary field of information visualization and data mining and data management that builds a bridge between the advantages of both human and computer to provide solutions for information overload. Visual analytics has two important focuses, visualization presentation and interactive techniques. Visual Representation is traditionally understood as an interface or view into data. Interaction is mainly about how the analyst requests new visual representations of data. It allows users consider different perspectives on the same information and provides the channels for users to interact with the different outputs.

Without interaction mechanisms that let users explore data, visualization tools can not maximize human capabilities to perceive and understand complex and dynamic data. However, interaction theory is much less mature than visual design theory, as from literatures we can see interaction techniques have not gain equal attention as visualization as most researchers tend to focus on visual representation in past decade [6]. The datasets presented in visualization in real-world applications are often very large with thousands or even millions of items, such as research citation and collaboration networks. Space-filling visualization is considered to be a good approach for visualizing large hierarchical datasets with better presentation of global patterns of the overall data structure. It has a high capacity for the visual representation of very large hierarchical data with excellent space efficiency. However, there are still many navigation problems existing in large scale datasets. This paper is an attempt to further explore the interaction theory based on navigation methods in space-filling and a usability study.

\section{Related Work and Motivation}

Space-filling visualization technique ensures space efficiency by dividing the display area into nested rectangles [3, 6, 16], polygons [4], ovals [5] or other shapes [11] and assigning them as geometrical regions to subsets of the entire dataset for displaying. Space-filling techniques, especially Treemaps and variations $[3,6,16]$, have also shown high applicability and commercial value in many areas such as finance analysis [7], sport reporting [8], image browsing and software and file system analysis $[9,10]$.

Although the layouts generated in space-filling visualization are very efficient in terms of space utilization, the issues of "view-ability" to produce userfriendly interactive interfaces and the ability to explore data accurately are critical, especially for visualizing large and deep relational datasets. This is because in 
space-filling visualizations as well as other visualization techniques, it is hard to discern between nodes and edges, hierarchical levels, labels and other properties when thousands of items in datasets are displayed concurrently. Therefore, an efficient and effective navigation scheme, combined with a visualization which provides users with necessary knowledge where to go is essential when navigating large data structures. The navigation scheme should enable users to interactively adjust views to reach the final view of a sub-graph, allowing them to obtain an optimal understanding of the data items and surrounding relational structure they are currently interested in, with minimal navigation steps.

The most commonly used interactive navigation technique in space-filling visualization is drilling-down + semantic-zooming [17], a quick and simple navigation scheme that enlarges the display of a specific portion of the graph allowing users to view the detail of this substructure of the graph. The typical user interaction for locating a node is clicking on a selected node and moving to the sub-structure rooted and its sub-structure and users can recursively select another sub-graph or tree until reaching the final substructure that contains the target node. This form of interaction is analogous to zooming into a region of interest with each step of the zoom operation being a sub-structure in the hierarchy.

However, under this navigation scheme, users are usually provided with very limited knowledge about where to go and where they have been by extracting contextual information from only one view. Users could spend a significant amount of time browsing for specific items in hierarchies because user has to frequently reform or re-establish their mental map of the relational structure to determine where they have been and where they should to go to find specific it. With the drill-down approach the number of wasted actions, a user may take to reach the specific item could be significantly increased because in this approach, traversing each successive layer requires abandoning the previous views. This can lead to disorientation during navigation and reduce the amount of contextual information available for the decision making.

Alternative space-filling techniques focus+context viewing (Single-Context View) provides users with a detail view of a focused sub-graph as well as a global view of the overall graph to maintain user orientation. Typical techniques include Sunburst [5], Information Slices [11], Fisheye + Zooming [12], Layering + Transparency [13] and Multiple Views [14].

However, in real-world, especially for financial data, interactive exploration could more than 10 hierarchal levels or exploration. The display of one current view and one or two context views [18] could still be insufficient for users to gain enough contextual information to make their further decisions on where they should go next.

To solve this problem, a new interaction method called multi-context visualization has been proposed for the navigation of large information. Instead of displaying a single or small number of context views as in traditional approaches, we provide users with the display of a progressive sequence of context views attempting to maintain the full contextual information. Comparing current mechanisms, full-Context view can enable users to interactively adjust views to reach the final view of a sub-graph, allowing them to obtain an optimal understanding of the data items and surrounding relational structure. This also allows users to trace each step of their interaction and makes it easy for them to jump or return to any level of the hierarchy they have already visited. Wong et al. [15] found that analysts will often look over the full structure of a semantic graph and mentally partition the graph into natural clusters of high activity or dense Sub-graphs. For example, after one target dataset has been characterized, the analyst may have to return back to larger view of picture to examine the relationship between the target and whole organization. The science of human concept and research implies that the display of rich context information produced in the exploration path could greatly increase the accuracy of user decisions and reduce the unsuccessful trips and unnecessary views during visual exploration of large hierarchies.

To subjectively prove the usefulness of context information of interactive visualization, this paper is to evaluate the full-context view visualization by conducting an experiment-based user study. The motivation of this usability study is to assess the effectiveness and efficiency of the chain-context view, by measuring the user performance, and to answer the question. Specifically, to answer whether more contextual information assists in making more accurate and easier decision, this usability test was designed to test if context-chain view has solved the most navigation problems in deep and large relational datasets. The evaluation of this usability study will provide further insight on possible improvements for existing interactive techniques to bridge the gap between interactive techniques and human analytical reasoning in applying visual analytics.

In following sections, a usability study will be conducted by comparing user performance with multicontexts, one context, and no context. Section 4 interprets and evaluates the results and discusses the further improvements. Finally, the conclusions will be drawn in Section 5.

\section{Usability Study}

In this section, it will first describe three compared interactive visualization techniques with multi-contexts, single context and no context, and then review the usability study including its user groups, and user tasks.

\subsection{Description of compared interactive navigation techniques}

There are two common interactive navigation techniques: Drilling-down + Semantic Zooming and Focus and Context Viewing. 
3.1.1 Drilling-down + semantic zooming This is the most commonly used interactive navigation technique. This quick and simple navigation scheme is widely established in current operation systems. Three steps in location or searching an object are:

1) Perceive relational structure from the view

2) Make decision about where to go

3) Click on a selected objective and move to the substructure until locate the target.

3.1.2. Focus and context viewing This is an alternative technique, provides users with a detail view of a focused sub-graph and overall graph to maintain user orientation. Focus and context viewing has already well developed into many applications in medical, engineering and financial area. Three steps in location or searching an objective are:

1) Perceive relational structure from the both views or a blended view

2) Make decision about where to go based on the knowledge gained from views

3) Click on a selected objective and move to next view until locate the target.

3.1.3 Multi-context Visualization This is the new method initially proposed by Huang and Nguyen for visual analytics in data mining [18]. Instead of displaying a single or two context views as in traditional approaches, it provides users with the display of a progressive sequence of context views, which only occupied a small portion of the display area. The animation techniques are applied to each interaction in order to maintain the user's mental orientation of views. Three steps in location or searching an objective are:

1) Perceive relational structure from the multicontents

2) Make decision about where to go based on the knowledge gained from whole context views and current view.

3) Click on a selected objective and move to any view from context views until locate the target.

The key question for this usability test is to find out whether the use of multiple contexts can provide efficient and effective visualizations? The usability study evaluates all three techniques using the same spacefilling layout algorithm and a drilling-down + semantic zooming navigation. There techniques are a) No-context view, b) Single-context view and c) Multi-context views or full-context views.

\subsection{User Group}

In this usability test, twelve subjects participated. They were undergraduates, postgraduates, and PhD students, and workers who use computers or deal with data analysis everyday. Their background varies vary from IT and mathematics and engineering and accounting. All were familiar with the concept of file and directory structures and had reasonable experience in performing standard file management routines. None had any previous experience using the navigation methods and were not familiar with space-filling concepts.

\subsection{User Tasks}

The user tasks involve minimum training and structured questionnaires with control experiments and a post-interview. In control experiments, there are questions with time limits and an open question in the end. There would be no demographic questions except Education background, in the structured interview.

3.3. 1 Procedures The introduction first was given by trainer to briefly describe the research and its reason, but in order not to influence their preference, the details of research were not to be informed. The training also included the introductions of system's features, interface and the structure of questionnaires and time for completing this survey. The candidates were informed to have about 10 minutes of training how to use the navigation systems prior to the experiment, and 5 minutes to read the tasks and notes in the questionnaires. Before starting the questionnaires, each subject was familiarized with both browsing tasks and navigation methods. During the questionnaires, they were allowed to give up or stop the trial, if the time exceeded 5 minutes in certain question. In the end, they were interviewed by the trainer about their preferences and suggestions.

3.3.2 Questionnaires The survey consists of 3 questionnaires separately for three methods a) Nocontext view, b) Single-context view and c) Multicontext views. Each questionnaire contains similar tasks. The difficulty of the tasks and hierarchy levels of the object increases along the way of answering the questions. Three types of tasks were designed to test the ability of the viewers to navigate through hierarchies, and they are finding nodes, returning to previously visited nodes, and listing all the ancestors of a node.

We designed 4 specific tasks to test an particular viewer's ability of navigating through a hierarchy; these include 1) with no specific-path pre-defined: this task requires the finding of a node at a specific level of the structure in which its path was unknown, 2) with specific path pre-defined: this task requires the finding of a node from a given path, 3) returning back to a visited node: this task requires the returning back to a previously visited context in which the node that has been visited in task 1 is found, and 4) listing all the ancestors of a node in the file hierarchy or indicating the correct path.

There were 7 questions in each experiment. Questions 1 and 4 required browsing with no specific path indicated (task 1), questions 2 and 3 required the finding of a target with a pre-defined path (task 2), question 6 required the returning back to a previously visited node (task 3 ), and question 5 required the listing 
of all the ancestors of a node (task 4). In the end, all participants were interviewed by the trainer about their preferences for each interactive visualization methods, i.e. question 7.

3.3.3 Control experiments To reduce learning effects, we used the three sets of hierarchies which were created with the same hierarchical structures but entirely different files. Participants were randomly assigned to one of three navigation methods first and continued experiments in one of three datasets in random order. Subjects performed the experiments on a 24 inches monitor with experimental window size of $1024 \times 768$ pixels and ran the prototype over windows XP. The tasks were explained to them before they began the trials. These results were obtained from these control experiments. In control experiments, we recorded whether the participant located the correct target, whether the participants withdrew from the questionnaires, whether the participants exceeded the time limit. The time was recorded for every question at all cases. In all, the whole control experiments involved: 12 participants $\mathrm{x} 3$ navigation methods (in 3 data sets) $\mathrm{x}$ 7 questions $=252$ trials and 12 participants $\mathrm{x} 3$ navigation methods $=36$ interviews.

\section{Evaluation}

In this section, it will evaluate the advantages of navigation methods, by performance measurement including efficiency and effectiveness and the assessment of confidence and preference, and finally it will discuss the lessons learned from this usability study and further improvements.

\subsection{Performance measurement description}

To measure the results, the research question contains two terms that need expanding.

4.1.1 Efficiency of visualization Efficiency of visual interactions can be measured by the numbers of unnecessary views in unsuccessful trips. During the navigation, an "unsuccessful trip" occurs when a user reaches the final view and still has not located the target. All the views including the final view during the browsing can be called as "unnecessary views". However, it would be unrealistic to measure the number of unnecessary trips during the interviews. In this usability study, the browsing time were measured for the candidates to locate the object.

Surprisingly, the outcomes of those first experiments showed that the performance in No-context and Singlecontext for task 1 to task 3 was overall better than and Multi-Context. This could be explained by the unfamiliarity of participants with the visualization at the first 15 to 20 minutes. Too much information and new interactive graphics might confuse users in the beginning. Therefore, with little training and experience, less complexity in the visualization and simple navigation scheme seemed to be a better choice for the browsing and navigation of large structure.

However, user performance improved much faster in multi-context visualization than other two techniques at the following experiments. Below is a summary of statistic results. From the table, we can see that full context navigation has comparable lower mean than other two navigation methods. It means full context navigation method is most efficient overall, especially when the participants are already familiar with the visualization.

4.1.2 Effectiveness of navigation Effectiveness is measured by evaluating if context information has enhanced the accuracy of users' decisions and confidence of making decisions. Accuracy can be measured by correctness rate of answers to questionnaires. From observation and interviews, we could see that most subjects expressed the frustration about finding a target in No-context and Single-context visualizations and more subjects found easily to give up the questionnaires in No-context and Single-context visualizations than Multi-context visualization. Most subjects gave the correct answers for the last three parent directories in the question 6 . The results prove that Nocontext view is the most ineffective navigation method. Conversely, it suggests that Multi-context visualization method can effectively support interactive visualization and reveal the data structure efficiently, as the experiments results showed that subjects completed several tasks faster and with fewer errors with full context. As we used small size dataset in experiments, it implied that the benefit of full context navigation will be amplified along with increasing density and complex of dataset.

Case Processing Sunary

\begin{tabular}{|c|c|c|c|c|c|c|c|}
\hline & \multicolumn{7}{|c|}{ Cases } \\
\hline & \multicolumn{3}{|c|}{ Included } & \multicolumn{2}{|c|}{ Excluded } & \multicolumn{2}{|c|}{ Total } \\
\hline & & N & Percent & $\mathrm{N}$ & Percent & $\mathrm{N}$ & Percent \\
\hline $\begin{array}{l}\text { Sum of Q1- Q6 } \\
\text { Navigation } \\
\text { Method }\end{array}$ & & 12 & $100.0 \%$ & 0 & $.0 \%$ & 12 & $100.0 \%$ \\
\hline \multicolumn{8}{|l|}{ Sun of $Q 1-06$} \\
\hline $\begin{array}{l}\text { Navigation } \\
\text { Method }\end{array}$ & \multicolumn{4}{|c|}{ Mean } & \multicolumn{3}{|c|}{ Std. Deviation } \\
\hline Full Context & \multicolumn{4}{|c|}{269.50} & \multicolumn{3}{|c|}{154.856} \\
\hline One Context & \multicolumn{4}{|c|}{316.00} & \multicolumn{3}{|c|}{186.221} \\
\hline No Context & \multicolumn{4}{|c|}{493.00} & \multicolumn{3}{|c|}{189.535} \\
\hline Total & & \multicolumn{3}{|c|}{430.60} & \multicolumn{3}{|c|}{192.012} \\
\hline
\end{tabular}

Figure1. Statistic Summary of All Questions 


\subsection{Confidence and preference}

We used preference section to reflect user's confidence for making better decision. In order to not influence user's preference, we asked half of candidates to start from No-context view and the other half start from Full-Context view method. In interviews, 10 out of 12 subjects chose full-context navigation as the preference over other two methods. Full context navigation method received the highest ratings for overall use. The overall pie graph showed the liker rating for full-context navigation design. About $42 \%$ subjects rated Multi-context visualization good barely, $8.3 \%$ subjects rated it very good. In summary, $75 \%$ subjects rated the design and usability for Multi-context navigation more than acceptable. (See Figure 2)

\section{3 Lessons learned from usability Study}

Since the usability Study started, there were a few improvements in datasets design and interface and features of navigation. For example, we increased the front and relocated the root from middle of screen to the left up corner to reduce the data cluster and confusion, and we also coloured the target to increase the visual interests for experiments. To remove the luck chance for finding a target, the new rules were gradually added into the experiments, for example, the subjects were not allowed to jump between questions, and had to answer the question in order. The browsing had to be conducted in right-clock wise.

However, to reduce the learning effect in three control experiments, we should increase the time for subject's familiarity and motivation to finish the questions. To increases the subjects' confidence about using interface, we should increase the training time at the same time.

\section{Conclusion}

To summarize, this usability study and evaluation proves that the use of more context information positively assists in making more accurate and easier decision. First, interaction mechanism with no context information negatively impacts user's performance to reestablish one's mental map to find the target. Second, the single context viewing interaction mechanism displays both focus and context simultaneously, which provides more accurate decisions on where they should go next. However, it is still insufficient for users in larger data sets. In contrast with the other two methods, full-context visualization effectively support interactive visualization and reveal the data structure efficiently and addressed the need of users to explore and browsing in details in large scale dataset. To conclude, multi-context visualization is a more efficient and effective navigation scheme to bridge gap between representation of focus and contextual data.
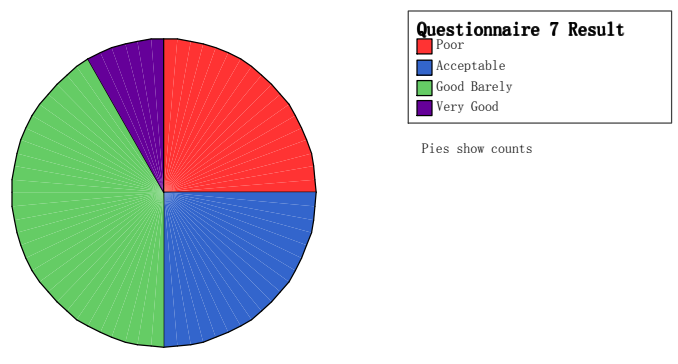

Pies show counts

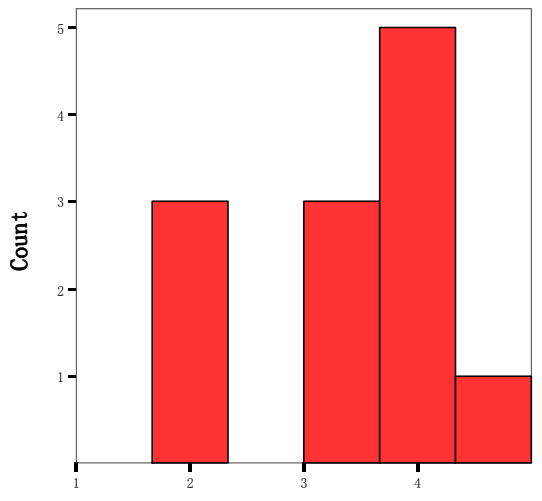

Questionnaire 7 Result

Questiornaire T Result- Liker Rank

\begin{tabular}{|l|l|l|l|l|l|}
\hline \multicolumn{2}{|l|}{} & Frequency & Percent & $\begin{array}{l}\text { Palid } \\
\text { Percent }\end{array}$ & $\begin{array}{l}\text { Cunulative } \\
\text { Percent }\end{array}$ \\
\hline Valid & Poor & 3 & 25.0 & 25.0 & 25.0 \\
\hline & Acceptable & 3 & 25.0 & 25.0 & 50.0 \\
\hline \multirow{2}{*}{ Good Barely } & 5 & 41.7 & 41.7 & 91.7 \\
\hline & Very Good & 1 & 8.3 & 8.3 & 100.0 \\
\hline & Total & 12 & 100.0 & 100.0 & \\
\hline
\end{tabular}

Figure2. Results of Question 7

\section{References}

[1] Thomas, J.J., Cook, K. A Visual Analytics Agenda. IEEE Computer Graphics and Applications, 26(5), pp. 10-13, 2006.

[2] Thomas, J.J., Cook, K. Illuminating the Path: Research and Development Agenda for Visual Analytics. IEEE Computer Society, 2006.

[3] Bederson, B.B., Shneiderman, B. and Wattenberg, M. Ordered and Quantum Treemaps: Making Effective Use of 2D Space to Display Hierarchies. ACM Transactions on Graphics, 21(4), pp. 833-854, 2002. 
[4] Balzer, M., Deussen, O. Voronoi Treemaps. In Proceeding IEEE Symposium on Information Visualization. IEEE, pp. 49-56, 2005.

[5] Stasko, J., Zhang, E. Focus+Context Display and Navigation Techniques for Enhancing Radial, SpaceFilling Hierarchy Visualizations. In Proceeding IEEE Symposium on Information Visualization. IEEE, pp. 5760, 2000.

[6] Bruls, M., Huizing, K., van Wijk, J.J. Squarified Treemaps. In Proceeding Joint Euro graphics and IEEE TCVG Symposium on Visualization, Springer, pp. 33-42, 2000.

[7] Jungmeister G.A., Turo, D. Adapting Treemaps to Stock Portfolio Visualization. Technical Report UMCPCSD CS-TR-2996, University of Maryland, College Park, Maryland 20742, USA, 1992.

[8] Jin,L., Banks, D.C. Hierarchical Visualization with Treemaps: Making Sense of Pro Basketball Data. IEEE Computer Graphics and Applications, 17(4), pp. 63-65, 1997.

[9] Balzer, M., Deussen, O., Lewerentz, C. Voronoi Treemaps for the Visualization of Software Metrics. In Proceeding ACM Symposium on Software Visualization, pp. 165-172, 2005.

[10] Baker, M.J., Erick, S.G. Space-filling Software Visualization. Journal of Visual Languages and Computing, 6(1), pp. 119-33, 1995.

[11] Andrew, K., Heidegger, H. Information Slices: Visualizing and Exploring Large hierarchies using Cascading, Semi-Circular Discs. In Proceeding LateBreaking Hot Topics-IEEE Symposium on Information Visualization, IEEE, pp. 9-12, 1998.

[12] Shi, K., Irani, P., Li, B. An Evaluation of Content Browsing Techniques for Hierarchical Space-Filling Visualization. In Proceeding IEEE Symposium on Information Visualization, IEEE, pp. 81-88, 2005.

[13] Nguyen, Q.V., Huang, M.L. A Focus+Context Visualization Techniques Using Semi-Transparency. In Proceeding $4^{\text {th }}$ International Conference on Computer and Information Technology, IEEE Computer Society, pp. 101-108, 2004.

[14] Baldonado, M.Q.W., Woodruff, A., Kuchinsky, A. Guidelines for Using Multiple Views in Information Visualization. In Proceeding Advanced Visual Interfaces (AVI'2000), ACM Press, pp. 110-119, 2000.

[15] Wong, P.C., Chin, G., Foote, H., Mackey, P., Thomas, J. Have Green: A Visual Analytics Framework for Large Semantic Graphs. In Proceeding IEEE Symposium on Visual Analytics Science and Technology, pp. 67-74, 2006.

[16] Johnson, B., and Shneiderman, B. Tree-Maps: A SpaceFilling Approach to the Visualization of Hierarchical Information Structures. In Proceeding 1991 IEEE Visualization, IEEE Computer Society, pp. 284-291, 1991.

[17] Baker, M.J. Eick, S.G. Space-filling Software Visualization. Journal Visual Languages and Computing, 6(1), pp. 119 - 133, 1995.

[18] Huang, M.L., Nguyen, Q.V. Context Visualization for Visual Data Mining. Visual Data Mining: Theory, Techniques and Tools for Visual Analytics, book chapter, to appear. 